\title{
Performance of the QWIP focal plane arrays for NASA's Landsat Data Continuity Mission
}

\author{
M. Jhabvala ${ }^{1}$, K. Choi ${ }^{2}$, A. Waczynski ${ }^{1}$, A. La $^{1}$, M. Sundaram ${ }^{3}$, E. Costard ${ }^{4}$, C. Jhabvala ${ }^{1}$, E. Kan ${ }^{1}$, \\ D. Kahle', R. Foltz', N. Boehm ${ }^{5}$, M. Hickey ${ }^{5}$, J. Sun ${ }^{2}$,

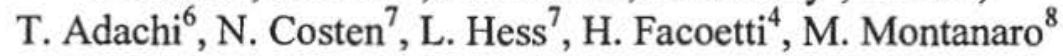 \\ 'NASA Goddard Space Flight Center, Greenbelt, Maryland 20771 USA \\ ${ }^{2}$ US Army Research Laboratory, 2800 Powder Mill Road, Adelphi, Maryland, 20783 USA \\ ${ }^{3}$ QmagiQ, LLC, 22 Cotton Road, Unit H, Suite 180, Nashua, NH 03063 USA \\ ${ }^{4}$ Thales Research \& Technology, 1 Avenue Augustin Fresnel 91767 Palaiseau Cedex, France \\ ${ }^{5}$ Global Science and Technology, 7855 Walker Drive, Greenbelt, MD 20770 USA \\ ${ }^{6}$ Catholic University of America, Washington DC 20064 USA \\ ${ }^{7}$ Muniz Engineering Inc., Seabrook, MD 20706 USA \\ ${ }^{8}$ Sigma Space Corp., Lanham, MD 20706 USA
}

\begin{abstract}
The focal plane assembly for the Thermal Infrared Sensor (TIRS) instrument on NASA's Landsat Data Continuity Mission (LDCM) consists of three $512 \times 640 \mathrm{GaAs}$ Quantum Well Infrared Photodetector (QWIP) arrays. The three arrays are precisely mounted and aligned on a silicon carrier substrate to provide a continuous viewing swath of 1850 pixels in two spectral bands defined by filters placed in close proximity to the detector surfaces. The QWIP arrays are hybridized to Indigo ISC9803 readout integrated circuits (ROICs). QWIP arrays were evaluated from four laboratories; QmagiQ, (Nashua, NH), Army Research Laboratory, (Adelphi, MD), NASA/ Goddard Space Flight Center, (Greenbelt, MD) and Thales, (Palaiseau, France). All were found to be suitable. The final discriminating parameter was the spectral uniformity of individual pixels relative to each other. The performance of the QWIP arrays and the fully assembled, NASA flight-qualified, focal plane assembly will be reviewed. An overview of the focal plane assembly including the construction and test requirements of the focal plane will also be described.
\end{abstract}

Keywords: QWIPs, quantum well detectors, infrared focal planes, IR detector arrays, GaAs detectors

\section{INTRODUCTION}

We have designed, fabricated, tested and fully space-flight qualified a QWIP-based focal plane assembly (FPA) for the joint NASA-US Geological Survey (USGS) Thermal Infrared Sensor instrument on the Landsat Data Continuity Mission (LDCM). The scheduled launch date is late 2012 and LDCM will continue the legacy of the NASA Landsat program. The mission science requirements were flowed down to the focal plane imposing strict performance metrics on the QWIP detector arrays. To ensure that these detector performance requirements were met, multiple development paths were pursued that involved four QWIP fabrication facilities; QmagiQ (Nashua, NH), Army Research Laboratory (Adelphi, MD), NASA/Goddard Space Flight Center (Greenbelt, MD) and Thales Research and Technology (Palaiseau, France). Since this represented the first time NASA was implementing a QWIP device in a critical earth science mission, it was and continues to be, essential that all risks be reasonably addressed and retired as early as possible in the program. Even though this development approach may seem like overkill, nothing is left to chance. The FPA required three $640 \times 512$ format QWIP arrays hybridized to Indigo ISC9803 readout integrated circuits (ROICs). Two fully qualified focal planes were developed and a third focal plane assembly was fabricated but not environmentally qualified. QWIP arrays were continuously fabricated, hybridized and tested for approximately 18 months. Over 70 potentially acceptable QWIP hybrids were fabricated and tested (and many more unused QWIP arrays were set aside) from all four laboratories. QWIP hybrids were produced by each laboratory that fully met the LDCM/TIRS mission requirements [1]. 
These requirements included pixel operability, spectral conversion efficiency (CE), dark current (at 43Kelvin), CE and dark current stability, read noise, full well capacity, power dissipation and thermal cycling and vibration survivability. A final discriminating metric was the pixel-to-pixel spectral response uniformity across a selected row of a given QWIP hybrid. It had always been a requirement that the pixel-to-pixel spectral uniformity be measured but was not intended to be used as selection criteria until an adequate pool of QWIP hybrids was successfully established. However, we did find a variation in the spectral uniformity of the QWIP arrays fabricated by the various laboratories. A detailed description of the TIRS instrument was presented in 2010 [2]. The instrument is required to monitor a wide range of environmental parameters from global climate change to agricultural water consumption. The selection of QWIP detector array technology was initially arrived at by necessity - other options were not feasible given the schedule, cost constraints and performance requirements imposed by the already-in-progress Landsat mission. However, as the concept phase evolved into the development, qualification and testing/performance phase of the TIRS instrument it became increasingly clear that the QWIP technology was uniquely suited for this (and other remote sensing) missions. The NEAT of the QWIP detectors was less than a tenth of what was required of the TIRS system, the technology was mature and widely available (relatively speaking), extremely reliable, large format arrays of a given spectral response were relatively easily produced and the format compatibility with existing commercially available ROICs was trivial [3]. This team came to the realization that where we have, for years (in some cases, decades), viewed and relegated QWIP devices as a niche technology [4] that when all things are considered QWIP technology can often be the preferred technology:

-the Technology Readiness Level (TRL) has been demonstrated

- relative ease of fabrication, rapid turn-around and availability

-physical realization of large format arrays

- precise spectral response selection over a very wide infrared range (from around $4 \mu \mathrm{m}$ to beyond $14 \mu \mathrm{m}$ )

-excellent NE $\Delta \mathrm{T}$

-excellent stability, uniformity and yield

-relatively low costs

-compatibility with existing cryocooler technology and;

- ease of operation

The successful development of this focal plane is largely attributable to the close and highly collaborative effort of engineers at Goddard, Army Research Lab, QmagiQ and Thales. Many arrays were fabricated and tested. However, the ultimate success lies in the ability to adjust process parameters to improve performance in an interactive and nimble fashion. This focal plane assembly was conceived and fully developed in slightly less than two years. Included in this time span is the:

- QWIP array design and mask generation

-QWIP array fabrication (over 200 arrays were fabricated)

-ROIC wafer procurement, indium deposition and bump patterning

-QWIP/ROIC hybridization

-Radiation, vibration and thermal qualification

-Entire focal plane design, fabrication and qualification (TRL-6)

-Complete characterization of over 70 flight candidates (all parameters)

-Engineering model focal plane assembly, characterization (containing 3 QWIP hybrids) and qualification

-Flight focal plane assembly, characterization (containing 3 QWIP hybrids) and qualification

-Flight spare focal plane assembly, characterization (containing 3 QWIP hybrids) and qualification

The focal plane performance requirements, assembly process, screening criteria and testing methodology of the QWIP array development for the Landsat/TIRS instrument will be described. Data will be presented on QWIP arrays produced by the various laboratories in support of this project.

\section{TIRS QWIP FOCAL PLANE REQUIREMENTS}

The QWIP array performance requirements are derived from the Landsat TIRS system requirements. For a review of the TIRS requirements see Reuter [5]. The TIRS instrument is required to perform a wide range of earth and atmospheric parametric measurements including evapotranspiration; cloud detection and analysis; mapping heat fluxes from cities; monitoring air quality; monitoring volcanic activity; monitoring the rain forests; biomass burning; industrial thermal pollution in the atmosphere, rivers and lakes; monitoring/tracking material transport in lakes and coastal regions; 
identifying insect breeding areas and medical applications. The QWIP array performance requirements are summarized in Table 1.

Table 1. Focal Plane Requirements

\author{
-Optical system \\ -Focal plane operating temperature \\ - Two $1 \mu \mathrm{m}$ wide spectral bands per array centered at \\ -Array format \\ -Pixel size \\ -Integration time \\ -Full well capacity \\ -Read noise \\ -Conversion Efficiency stability \\ $-\mathrm{I}_{\mathrm{D}}$ variation under stable operating conditions \\ -Power dissipation/array (4-outputs) \\ -Pixel-to-pixel spectral uniformity \\ -Co-planarity of the 3 QWIP arrays
}

f $/ 1.64$

$43 \mathrm{~K}$

$\mathrm{I} 0.8 \mu \mathrm{m}$ (Band 1), $12.0 \mu \mathrm{m}$ (Band 2)

$640 \times 512$

$25 \mu \mathrm{m} \times 25 \mu \mathrm{m}$

$5.5 \mathrm{~ms}$

$>5 \mathrm{Me}-$

$<1000 \mathrm{e}-$

$\pm<0.15 \% \sigma /$ mean over $35 \mathrm{sec}$

$<0.4 \%$ of mean over $45 \mathrm{~min}$

$<0.2 \%$ of mean over $45 \mathrm{~min}$

$<270 \mathrm{~mW}$

Measure and document

$< \pm 10 \mu \mathrm{m}$ within common focal plane

The relative spectral response measurement requirements are:

\begin{tabular}{|c|c|c|}
\hline Wavelength, $\lambda(\mu \mathrm{m})$ & $\underline{\Delta \lambda / \operatorname{step}(\mathrm{nm})}$ & Resolutio \\
\hline $1.8-7.0$ & 50 & 60 \\
\hline $7.0-10.0$ & 50 & 80 \\
\hline $10.0-10.5$ & 100 & 80 \\
\hline $10.5-12.3$ & 50 & 80 \\
\hline $12.3-13.0$ & 100 & 80 \\
\hline $13.0-15.0$ & 100 & 175 \\
\hline $15.0-20.0$ & 100 & 120 \\
\hline
\end{tabular}

The predominant detector noise source is the dark current fluctuation resulting from the thermal instability of the focal plane assembly. (The predominant system noise source is the noise resulting from the telescope temperature instability.) The TIRS system NE $\Delta T$ requirement is NE $\Delta T \leq 0.33 \mathrm{~K}$ for a $300 \mathrm{~K}$ source over all wavelengths from $10.5-12.3 \mu \mathrm{m}$ (not to be confused with the detector NE $\Delta \mathrm{T}$, which is much lower). Included in this TIRS system NEAT calculation are the noise contributions from:

- the QWIP dark current shot noise

- the noise resulting from the dark current variation due to temperature instability

- the ROIC read noise (<1000e-)

- the A/D quantization noise (<700e-)

- the added focal plane electronics noise $(<1000 \mathrm{e}-)$

- the shot noise from the target (300K)

- the noise resulting from the telescope temperature instability

- the noise resulting from the telescope mirror temperature instability

- the noise resulting from the telescope optics temperature instability

Implicit in these requirements is the ability to control the NEST by adjusting the QWIP dark current. A lower dark current will lead to a lower NE $\Delta T$. The focal plane temperature is assumed to be fixed at $43 \mathrm{~K} \pm 0.010 \mathrm{~K}$. However, both the dark current and the conversion efficiency are strongly dependant on the detector bias voltage and as a consequence a trade-off between these two parameters can be made to optimize the system NEAT. The NE $\Delta T$ can be expressed by:

$$
N E \Delta T=\left[\Sigma\left(I_{N}\right)^{2}+\Sigma\left(I_{D}\right)^{2}\right]^{1 / 2} / S_{\Delta T}
$$


In this case, $\Sigma I_{D}$ refers to the noise contributions from the dark current shot noise and the dark current fluctuation arising from the focal plane temperature instability and $\Sigma I_{N}$ is the noise contribution from all the other sources listed above. $S_{\Delta T}$ is the change in signal for a $1 \mathrm{~K}$ change in scene temperature and is directly proportional to the conversion efficiency;

$$
\mathrm{S}_{\Delta \mathrm{T}}=\mathrm{CE} \cdot \Omega \cdot \mathrm{t}_{\mathrm{int}} \cdot \mathrm{t}_{\mathrm{opt}} \cdot \mathrm{A}_{\mathrm{D}} \cdot \mathrm{Q}(\lambda)
$$

$\mathrm{S}_{\Delta \mathrm{T}}$ is the number of electrons generated per pixel for a $1 \mathrm{~K}$ change in source temperature

$\mathrm{CE}$ is the conversion efficiency

$\Omega$ is the solid angle viewed by the pixel

$\mathrm{t}_{\mathrm{nnt}}$ is the signal integration time

$t_{\text {opt }}$ is the complete optical transmission of the system

$A_{D}$ is the pixel area

$Q(\lambda)$ is the spectral photon flux from a source at temperature T in the spectral band $\lambda_{1}-\lambda_{2}$

The graph below in figure 1 illustrates the trade-off that can be made between a lower (or higher) dark current and a correspondingly lower (or higher) conversion efficiency. This CE- $I_{D}$ relationship is very important in selecting the operating point of the QWIP arrays. If it turns out that the cryocooler is struggling to meet the $43 \mathrm{~K}$ operating temperature then the dark current will rise. However, this dark current increase can be offset by reducing the detector voltage, which also leads to a reduction in CE but so long as the combination stays below the curve the Landsat science is not compromised.

CE vs Dark Current for NEdT < $0.33 \mathrm{~K}$ Integration time $=5.5 \mathrm{~ms}$

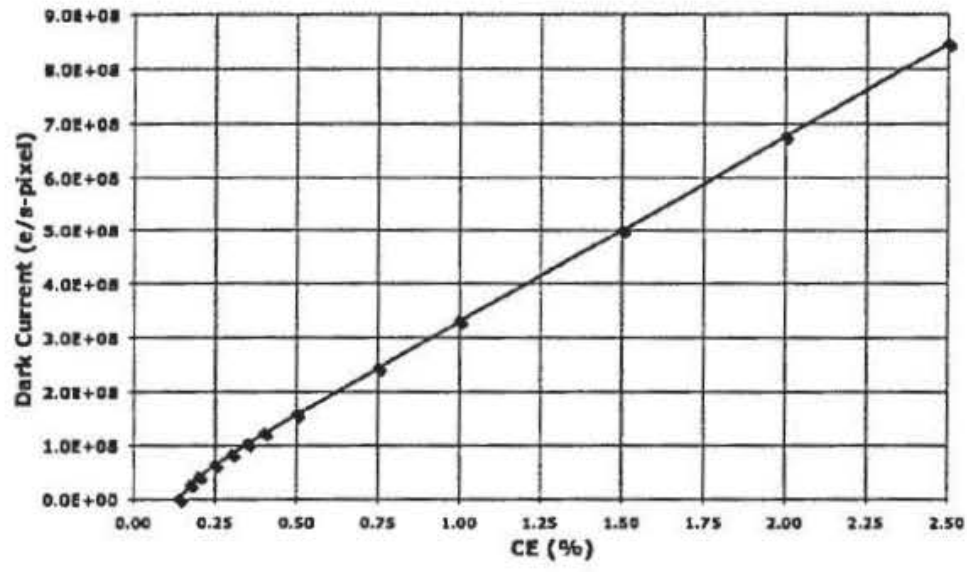

Figure 1. The trade-off between the QWIP detector dark current and conversion efficiency for NE $\Delta \mathrm{T} \leq 0.33 \mathrm{~K}$. Combinations below the curve meet the TIRS mission NEAT requirement.

\section{QWIP DETECTOR HYBRIDS}

The decision to utilize QWIP technology as the basis for a Landsat thermal imaging instrument was made very late in the LDCM development cycle. In order to minimize risk and maximize the chances for success a multi-pronged approach was pursued in the development of the QWIP arrays. Initially three groups, one at Goddard, one at ARL and one at QmagiQ each fabricated arrays. A fourth group, at Thales, also fabricated arrays in support of TIRS but they started about a year later. Goddard and ARL worked both independently and jointly in their fabrication efforts. In addition to the QWIP array fabrication, arrays from each source were hybridized in Goddard's Detector Development laboratory (the QmagiQ arrays were hybridized by both QmagiQ and Goddard). Subsequent array thinning was performed by each laboratory based on their own thinning recipes. The QWIP array design started in September 2008 and the fabrication started in January 2009. In order to meet the broad spectral response requirements of the mission some experimentation with the superlattice growth recipe was necessary. Ultimately, the aluminum mole fraction, $\mathrm{x}$, in the $\mathrm{Al}_{\mathrm{x}} \mathrm{GaAs}_{1-\mathrm{x}} / \mathrm{GaAs}$ structure was $<17 \%$. Scanning electron microscope images of the surface of each type of QWIP array are shown in figure 2. QmagiQ, LLC, fabricated the grating QWIP array and the corrugated QWIP (C-QWIP) 
was jointly fabricated by ARL and NASA/GSFC. The ROICs required backside lapping and patterning which was performed by the Goddard team prior to wafer dicing.
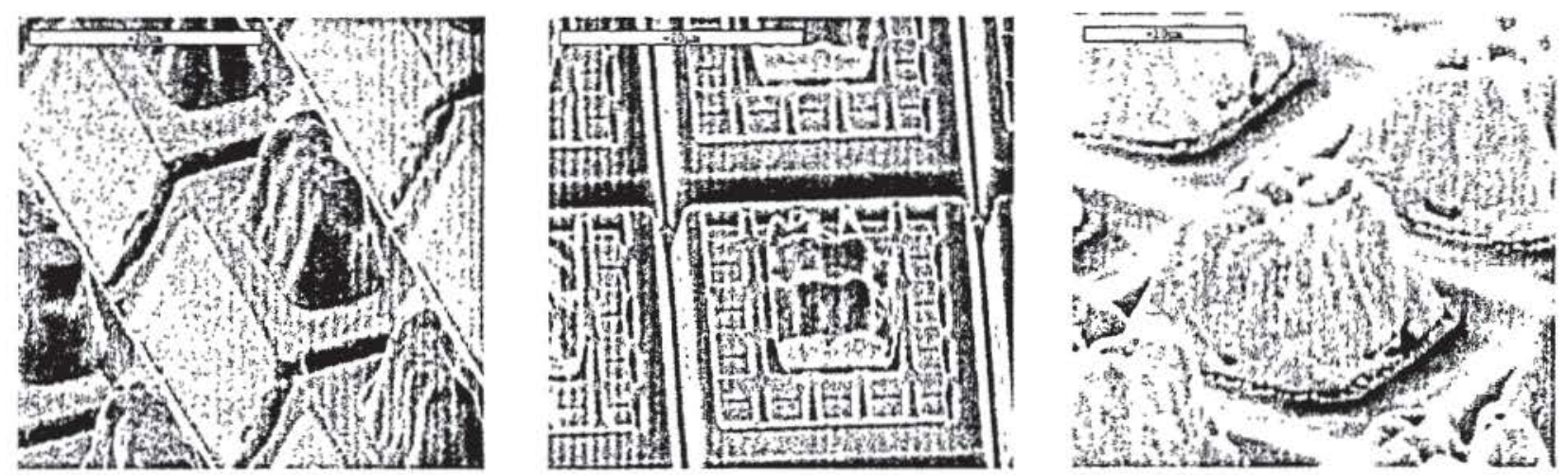

Figure 2. Scanning electron microscope images of the C-QWIP (left), the grating-based QmagiQ QWIP surface (center) and the Thales grating-based QWIP (right).

Table 2 provides a general summary of the volume of array processing that was performed in support of the TIRS project. Not included in the table is the post-processing of the Indigo 9803 ROIC wafers necessary for the hybrid alignment onto the silicon carrier. Since the focal plane required three QWIP hybrids, or sensor chip assemblies (SCAs) that are precisely aligned in the $\mathrm{x}, \mathrm{y}$ and $\mathrm{z}$ directions, alignment marks were required on the back of each ROIC die. Additionally, to ensure height uniformity (all 3 arrays must lie at the same focal point), the ROIC wafers were uniformly thinned and polished to $720 \mu \mathrm{m} \pm 2.5 \mu \mathrm{m}$.

Table 2. Sum mary of QWIP Array Fabrication

\begin{tabular}{|c|c|c|c|c|}
\hline QWIP Arrays & Goddard/ARL & QmagiQ & Thales & Totals \\
\hline Number of GaAs wafers processed & 7 & 5 & 3 & 15 \\
\hline Potential arrays/wafer & 16 & 16 & 8 & 216 \\
\hline Number of arrays hybridized & 22 & 41 & 3 & 66 \\
\hline Number of failed SCAs (damaged) & 2 & 4 & 0 & 6 \\
\hline Number of SCAs tested & 20 & 37 & 3 & 60 \\
\hline Number of SCAs meeting TIRS spec & 13 & 21 & 3 & 37 \\
\hline Number of engineering grade (fully functional) & 7 & 16 & N/A & 23 \\
\hline Functioning QWIP/ROIC SCA yield & $20 / 22=90.9 \%$ & $37 / 41=90.2 \%$ & $3 / 3=100 \%$ & $60 / 66=90.9 \%$ \\
\hline QWIP/ROIC SCA yield to TIRS specifications & $13 / 22=59.1 \%$ & $21 / 41=51.2 \%$ & $3 / 3=100 \%$ & $37 / 66=56.1 \%$ \\
\hline
\end{tabular}

The focal plane assembly is shown in figure 3 with and without the filters. Each QWIP SCA has a filter element spectrally centered at 10.8 and $12.0 \mu \mathrm{m}$ with a designed spectral width of $1.0 \mu \mathrm{m}$. The filter elements each span the entire SCA and are 32 rows wide. The TIRS science requires that all pixels in at least one row in each band fully meet the technical specifications. If there are no "perfect" science rows it is also acceptable to utilize pixels from two rows in a given band to artificially create a single perfect row. A key requirement in the construction of the focal plane is ensuring that the photo responsive regions of each QWIP array are vertically aligned to within $10 \mu \mathrm{m}$. There are multiple epoxy interfaces between the invar baseplate and the hybrids in addition to the standard machining and assembling tolerances one can reasonably expect to achieve. 

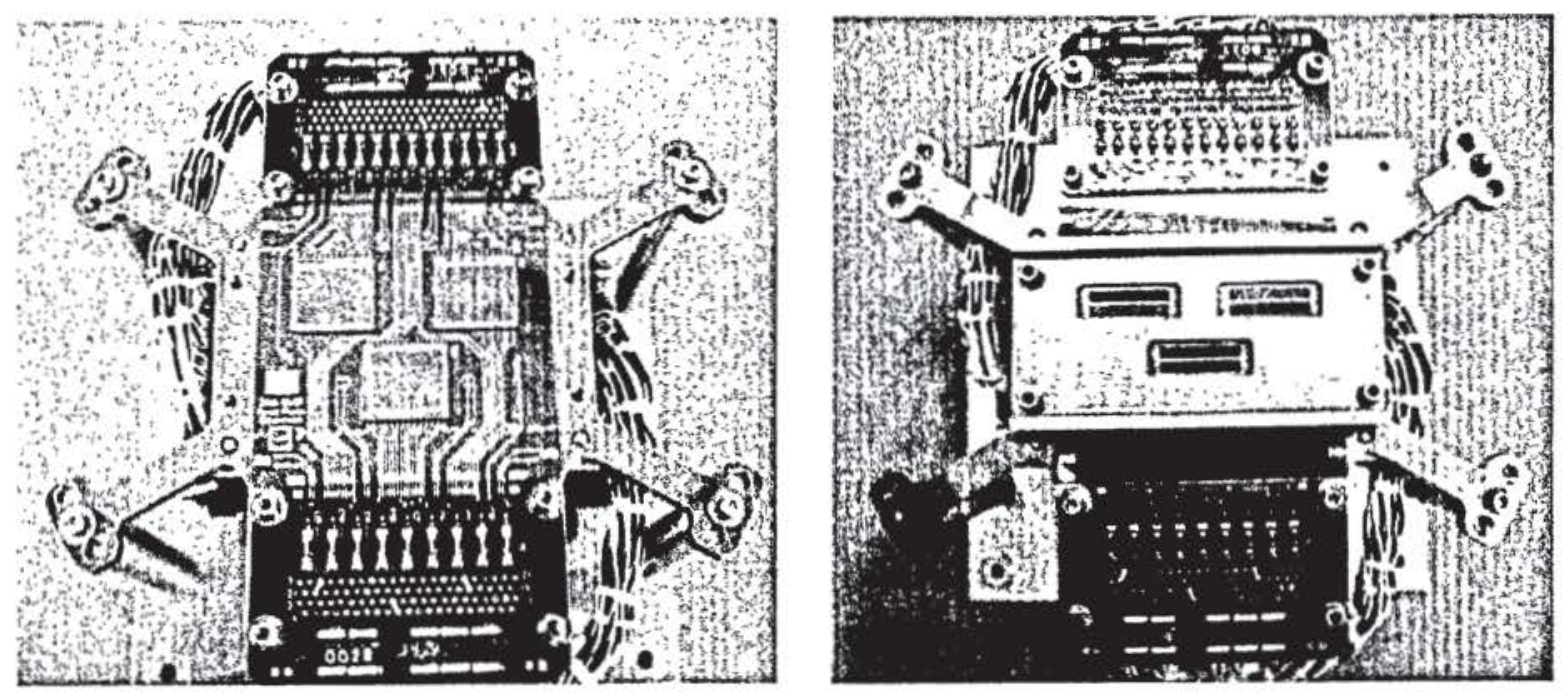

Figure 3. The TIRS focal plane. Left; photograph of the focal planes with the three QWIP SCAs exposed. Right; the FPA with the filter assembly installed.

The steps in the assembly process are:

- Indium bump bond the QWIP array to the ROIC and epoxy underfill

- Mechanically thin the GaAs QWIP surface to $\sim 40 \mu \mathrm{m}$

- Remove remaining GaAs

- Epoxy the silicon carrier substrate to the invar baseplate with $80 \mu \mathrm{m}$ diameter micro-bead spacers

- Epoxy the QWIP hybrids to the silicon substrate with $20 \mu \mathrm{m}$ diameter micro-bead spacers

- Perform wire bonding of the ROIC to the silicon substrate

- Install and wire bond temporary connector boards (known as daughter boards)

- Perform room temperature functionality tests after each hybrid is attached

- Replace temporary daughter boards with flight daughter boards

- Wire bond silicon substrate to daughter boards

- Perform profilometer measurements of the focal plane surface

- Perform comprehensive performance test of the focal plane assembly

- Install filter assembly

Shown in figure 4 is a profilometer image of the assembled FPA illustrating the surface height variance. The arrow identifies the maximum vertical distance between all three SCAs (which was entirely contained in the one SCA). This distance was determined to be I7.08 $\mu \mathrm{m}$, corresponding to $\mathrm{a} \pm 8.54 \mu \mathrm{m}$ deviation from a common virtual plane (the actual focus of the telescope).

\section{QWIP HYBRID TEST RESULTS}

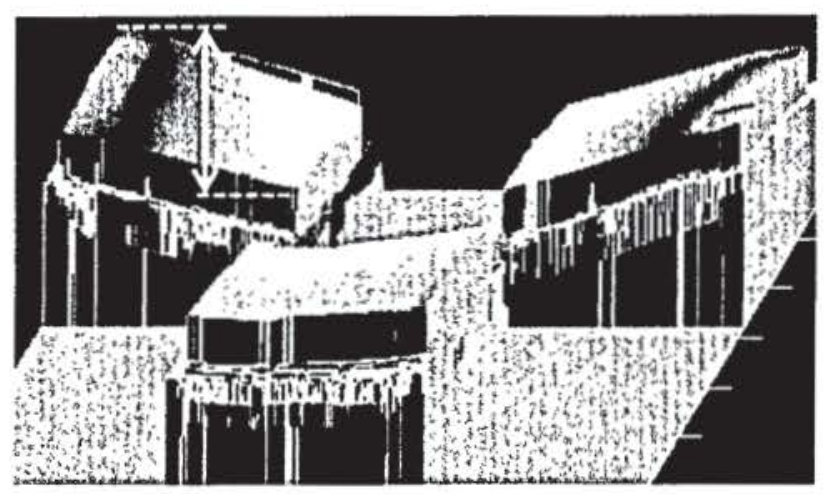

Figure 4. A ZYGO profilometer scan illustrating the surface height variation between the $3 \mathrm{SCAs}$. The largest variation occurred entirely in the top left hybrid.

The assembled QWIP hybrids are subjected to multiple testing cycles. The QWIP hybrid is temporarily bonded into an 84-pin leadless chip carrier (LCC). The first test is a ROIC go/no-go aliveness test at room temperature. Following this test the SCA is installed into a quick turn-around time cryo-cooled camera test system and the hybrid is subjectively 
analyzed for suitability. Infrared images are taken with the SCA at $\sim 43 \mathrm{~K}$ and obvious flaws such as epoxy voids, clusters of dead or hot pixels, line outages, etc. are surveyed. If the SCA looks promising it is then transferred to a calibrated test system (the complete cycle time for this "quick-look" test is less than 4 hours). A sample of one of these images is shown in figure 5. Candidate detector assemblies are then tested while still in the LCC configuration. The tests performed include (in approximate order):

- Signal versus integration time (linearity)

- Dark current vs. detector bias voltage and temperature

- Relative spectral response

- Absolute conversion efficiency versus wavelength

- 45-minute dark current stability

- 45-minute absolute CE stability

- Noise performance

- Noise equivalent delta temperature (NE $\Delta \mathrm{T})$ compliance

- Non-functional pixels

- Full well compliance

- Row selection for TIRS science

- Power dissipation

- Spectral uniformity

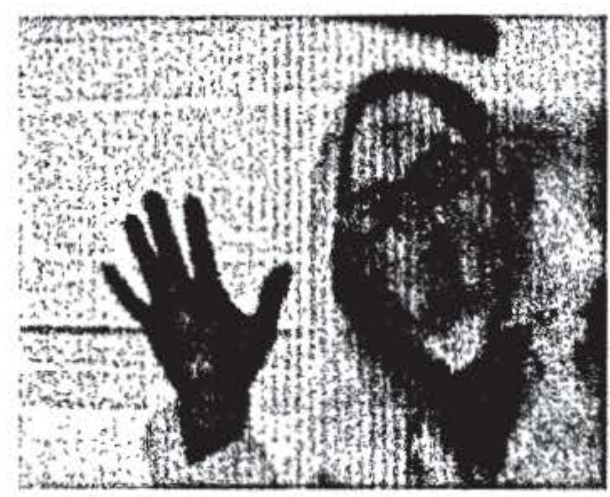

A final analysis of the test data is performed using the TIRS noise model

Figure 5. A "quick-look" IR image of a flight candidate SCA. This SCA was ultimately used in the TIRS focal plane assembly. to determine the SCA operating point to meet the TIRS system NEAT requirement (detector bias voltage selection, ROIC gain). Test results will be presented for science grade (best quality) QWIP arrays fabricated by the QmagiQ, Goddard/ARL and Thales groups. Each group designed and fabricated the QWIP arrays based on their established processes. Both the QmagiQ and Thales arrays implement a grating structure for optical coupling (no anti-reflection coating was applied to either version). The Goddard/ARL QWIP arrays utilized the corrugation method to provide the optical coupling. Either approach is transparent to the TIRS misșion as long as the performance requirements are satisfied. The indium deposition, patterning, bump bonding, epoxy backfilling and wire bonding occurred mostly at Goddard, however, QmagiQ thinned and wire bonded their own devices.

\subsection{Linearity and dark current measurements}

The linearity of the ROIC (set to a gain of 1.33) over the entire dynamic range is shown in figure 6. It is measured by integrating dark current and monitoring the output voltage of the ROIC (converted to digital numbers, DNs). There are essentially two linear regions. The low signal $(<20,000$ counts) conversion is $\sim 33 \mathrm{e}-/ \mathrm{DN}$ while the higher signal conversion is $\sim 100 \mathrm{e}-/ \mathrm{DN}$. The ROIC maximum output signal corresponds to $\sim 11 \mathrm{Me}$-. The dark current was measured as a function of temperature and detector bias. The plots shown in figure 7 are for three temperatures around the TIRS operating temperature of $43 \mathrm{~K}$. The dark current is typically less than $1 \mathrm{E} 8 \mathrm{e}-/ \mathrm{s}$ or $2.6 \mu \mathrm{A} / \mathrm{cm}^{2}$ and at $43 \mathrm{~K}$ can be as low as $0.12 \mu \mathrm{A} / \mathrm{cm}^{2}$ under reasonable operating conditions. One of the major noise sources in the TIRS instrument is the dark current fluctuation arising

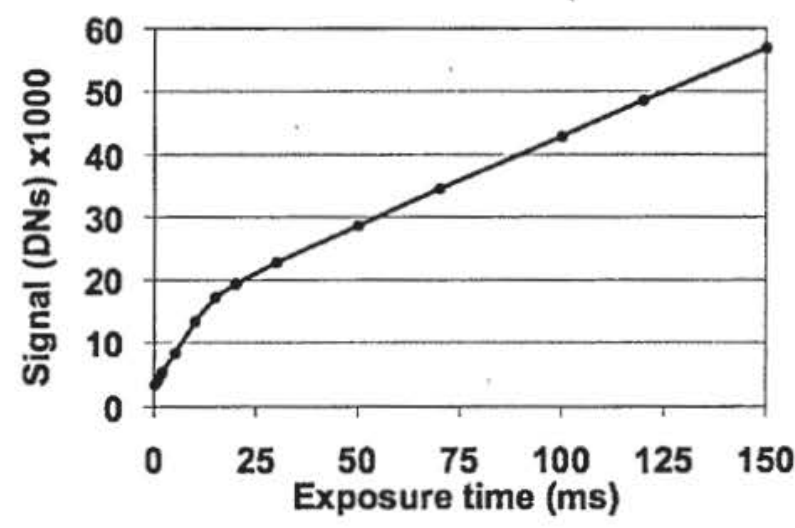

Figure 6. The ROIC output as a function of the QWIP integration time.

from the focal plane temperature instability. If the focal plane can be cooled to $41 \mathrm{~K}$ then this plane temperature instability becomes a negligible noise source. 

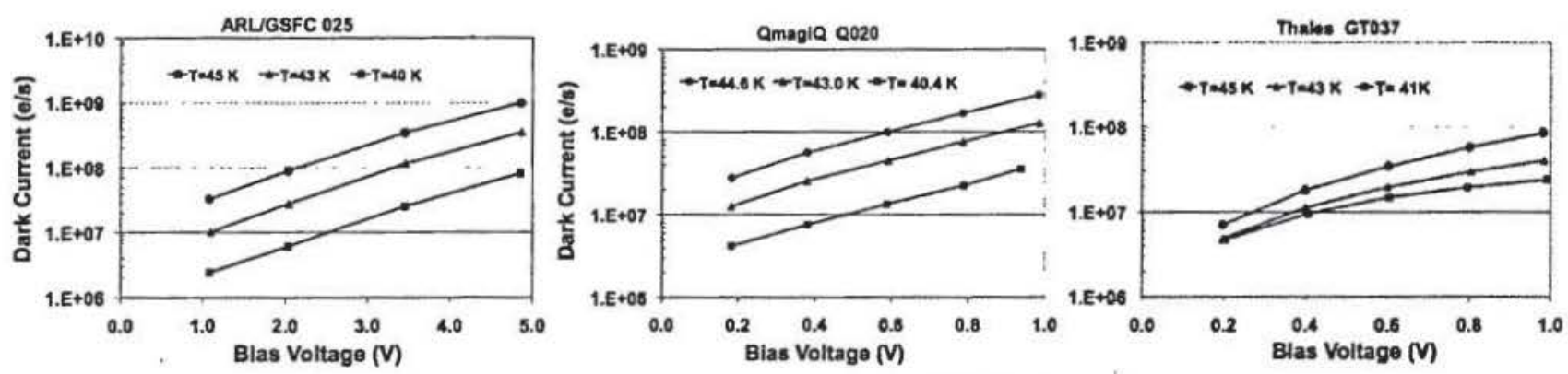

Figure 7. Dark current vs bias voltage at 3 temperatures at and near the TIRS FPA expected temperature of $43 \mathrm{~K}$.

\subsection{Spectral response and absolute conversion efficiency}

The relative spectral response is measured using a SpectralPro $300 \mathrm{i}$ monochromator. The spectral measurements are taken with $80 \mathrm{~nm}$ resolution over the 7-15 $\mu \mathrm{m}$ band. The sampling in the $10-13 \mu \mathrm{m}$ region (TIRS inband) is every $0.05 \mu \mathrm{m}$ and in the $7-10$ and $12-15 \mu \mathrm{m}$ (outof-band) the spectrum is sampled at $0.5 \mu \mathrm{m}$ intervals. The array is then exposed to an unfiltered $800^{\circ} \mathrm{C}$ blackbody source and the pixel signal is recorded. This signal is the product of the QWIP relative response and the $800^{\circ} \mathrm{C}$ blackbody Planck function. By dividing the pixel signal by the calculated spectral response $\mathrm{x} 800^{\circ} \mathrm{C}$ Planck function gives the absolute $\mathrm{CE}$. This measurement is performed on "each array at different detector bias voltages. It was determined from experiments performed early in the. program that the absolute $\mathrm{CE}$ was insensitive to small temperature variations. We therefore cool the detector a few $\mathrm{K}$ below the $43 \mathrm{~K}$ required operating temperature to eliminate dark current effects. The arrays from the different laboratories each require unique bias voltages to optimize the conversion efficiency-dark current trade-off. Shown in figure 8 is the absolute conversion efficiency versus wavelength for the same arrays as shown in figure 7 above. Each laboratory obtained their own GaAs wafers with the epitaxially grown superlattice defined by their own recipes, which explains the difference in spectral response from one lab to another. It is clear that the $\mathrm{CE}$ increases with increasing detector bias voltage. That is also the case with dark current. Increasing $\mathrm{CE}$ increases the optical signal conversion but with an increase in dark current shot noise and an increase in the noise due to dark current fluctuations with temperature. For optimum system NE $\Delta \mathrm{T}$ performance a typical compromise is to select a detector bias voltage that is $\sim 80 \%$ of the maximum. However, once in orbit the detector bias can be remotely accessed and if the dark current is lower than anticipated (that is, the TIRS FPA is operating colder than expected) the detector bias can be increased although the $\mathrm{S} / \mathrm{N}$ limitation will most likely be due
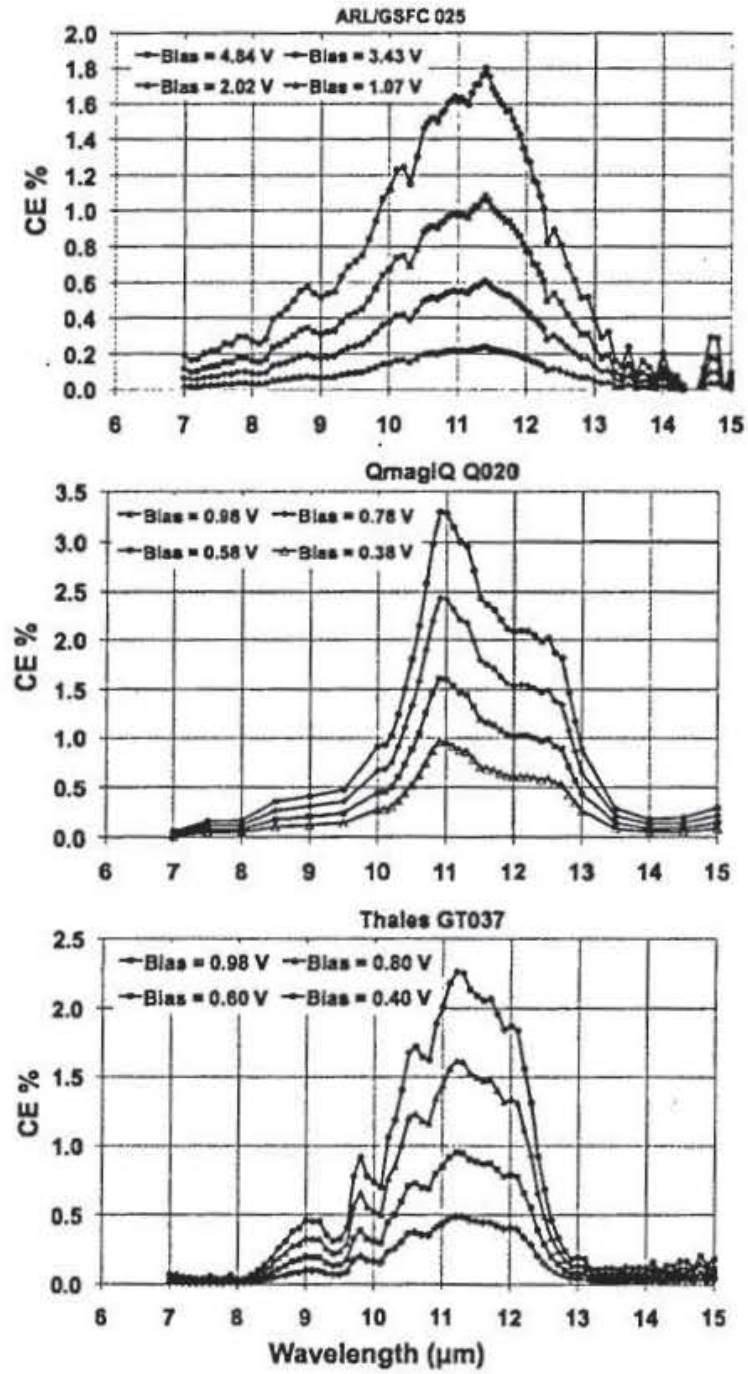

Figure 8. CE vs wavelength with different bias voltages. 
to sources unrelated to the detectors.

\subsubsection{Filter design and spectral response}

Once the spectral response has been determined, the spectral transmission of the $10.8 \mu \mathrm{m}$ and $12.0 \mu \mathrm{m}$ filter elements is specified. The goal is to tune the filter response such that the combined QWIP response/filter transmission approximates a $1 \mu \mathrm{m}$ uniform band pass combined response. Since the spectral responses are different for each of the arrays fabricated with a different superlattice growth recipe, the filters are customized to specific QWIP arrays. The combined filter/QWIP response for the QmagiQ process is shown in figure 9. The two plots are for a single pixel in two rows-one pixel under each filter element. The important characteristic is the spectral shape. The QmagiQ arrays were selected because of their extremely consistent pixelpixel spectral response (see section 4.4). It is important to emphasize that the filters were fabricated specifically to modify the spectral response of the QmagiQ arrays whose spectral response is shown in figure $8(\mathrm{Q} 020)$. To produce a similar combined response with either the ARL/Goddard or Thales arrays would require entirely different filters. The flight spare unit will have filters with a different transmission characteristic than the flight unit.

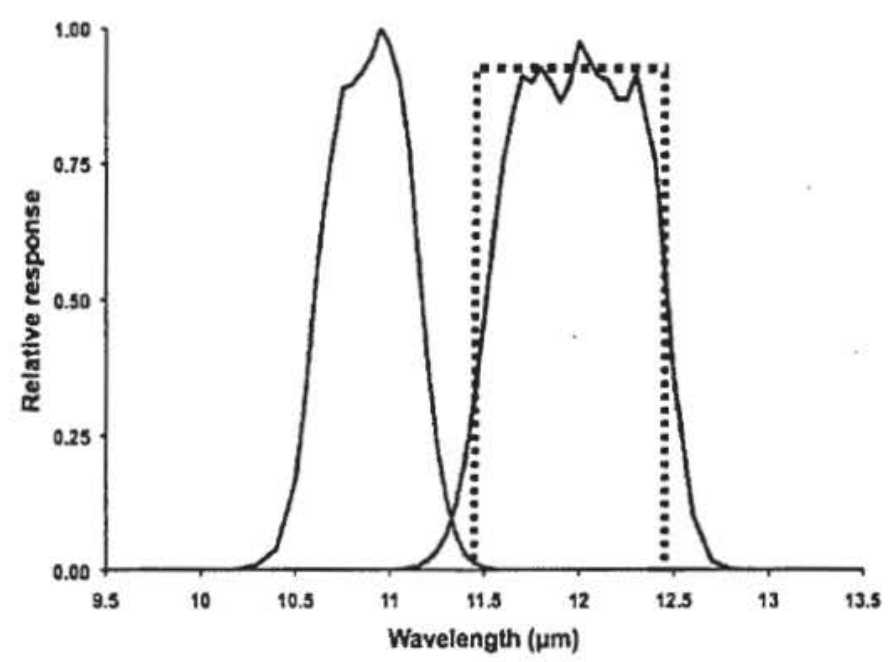

Figure 9. The combined filter/QWIP array spectral response of two pixels--one in each filter band. The goal is to make the combined response close to a $1.0 \mu \mathrm{m}$ band pass as illustrated by the dotted lines for the $12.0 \mu \mathrm{m}$ band. The pixel spectral response is identical to Q020 shown in figure 8 prior to filtering. The array was fabricated by QmagiQ.

\subsection{Dark current and conversion efficiency stability}

The intrinsic stability of the detector technology determines how often the system must be recalibrated in orbit. The more stable the detector the less frequently calibration is required. Two parameters were evaluated for stability; dark current and conversion efficiency. The stability of the dark current is measured by collecting a dark image every 2.5 minutes over a 45-minute time interval. To calculate the change in dark current we measured the maximum and minimum dark current values for every pixel in the entire array over the 45 -minute period. The TIRS requirement is that this maximum variation be $<5.1 \mathrm{E} 5 \mathrm{e}-/ \mathrm{s}$. The conversion efficiency stability is measured by exposing the array to a $315 \mathrm{~K}$-blackbody source and monitoring the signal over a 45-minute time interval. The blackbody source is under vacuum to eliminate the ambient effects. Ninety frames are collected every 1.5 minutes for 45 minutes and averaged to yield a single image frame. The variation of every pixel signal in this frame (which is obtained every 1.5 minutes) is evaluated over the 45 minutes and the TIRS requirement is that the CE of every pixel should not change by more than $0.4 \%$ in 45 minutes. A tabular summary of the stability performance measured against the TIRS requirements is shown below in the three tables. Also included in the tables are the number of pixels with excess dark current, too low CE and non-functional pixels. The thresholds for the different arrays are determined from the TIRS noise model and the selected operating conditions for that array. The striking conclusion is that based on these parameters all three of these arrays easily meet the science requirements for the TIRS mission. Each of the arrays identified in the tables below also met all the other requirements identified in Table 1 (such as the full well, read noise, power dissipation and other metrics). Given that TIRS requires only a few perfect rows (and that to obtain a perfect row, two imperfect rows can be utilized) all these arrays meet this requirement by two orders of magnitude! The challenge for the science team was to select arrays for the mission and since each technology appears to be over qualified an additional test was performed that would help discriminate one detector array from another. However, it must be emphasized that based on the mission specifications, arrays of each variety were and are flight worthy. 
QmagiQ Q020

\begin{tabular}{|c|c|c|c|}
\hline Type of Pixel & Thresholds & Total & $\%$ of Array \\
\hline Hot & $\mathrm{I}_{\mathrm{d}}>8.394 \times 10^{7} \mathrm{e}-/ \mathrm{s}$ & 24 Pixels & $0.007 \%$ \\
\hline Low CE & $\mathrm{CE}<0.30 \%$ & 19 Pixels & $0.006 \%$ \\
\hline Unstable Dark Current & Change in $I_{d}>5.1 \times 10^{5} \mathrm{e}-/ \mathrm{s}$ over 45 minutes & 17 Pixels & $0.005 \%$ \\
\hline Unstable CE & Change in $\mathrm{CE}>0.4 \%$ of mean over 45 minutes & 46 Pixels & $0.014 \%$ \\
\hline Total Non-functional Pixels & $\mathrm{NE} \Delta \mathrm{T}>0.33 \mathrm{~K}$ & 89 Pixels & $0.027 \%$ \\
\hline Total Functional Pixeis & $\mathrm{NE} \Delta \mathrm{T} \leq 0.33 \mathrm{~K}$ & 327,591 Pixels & $99.94 \%$ \\
\hline Total Perfect Rows & Row containing 640 functional pixels & 433 Rows & $84.57 \%$ \\
\hline
\end{tabular}

\section{ARL/GSFC 025}

\begin{tabular}{|l|l|l|l|}
\hline \multicolumn{1}{|c|}{ Type of Pixel } & \multicolumn{1}{c|}{ Thresholds } & \multicolumn{1}{c|}{ Total } & \multicolumn{1}{c|}{$\%$ of Array } \\
\hline Hot & $I_{d}>5.04 \times 10^{7} \mathrm{e}-/ \mathrm{s}$ & 30 Pixels & $0.009 \%$ \\
\hline Low CE & CE $<0.22 \%$ & 75 Pixels & $0.022 \%$ \\
\hline Unstable Dark Current & Change in $1_{d}>5.1 \times 10^{5}$ e-/s over 45 minutes & 48 Pixels & $0.014 \%$ \\
\hline Unstable CE & Change in CE $>0.4 \%$ of mean over 45 minutes & 89 Pixels & $0.027 \%$ \\
\hline Total Non-functional Pixels & NEdT $>0.33 \mathrm{~K}$ & 169 Pixels & $0.051 \%$ \\
\hline Total Functional Pixels & NEdT $\leq 0.33 \mathrm{~K}$ & 327511 Pixels & $99.87 \%$ \\
\hline Total Perfect Rows & Row containing 640 functional pixels & 408 Rows & $79.68 \%$ \\
\hline
\end{tabular}

Thales GT037

\begin{tabular}{|l|l|l|l|}
\hline \multicolumn{1}{|c|}{ Type of Pixel } & \multicolumn{1}{c|}{ Thresholds } & \multicolumn{1}{c|}{ Total } & \multicolumn{1}{c|}{$\%$ of Array } \\
\hline Hot & $\mathrm{I}_{\mathrm{d}}>\mathbf{5 . 0 4 \times 1 0 ^ { 7 } \mathrm { e } - / \mathrm { s }}$ & 0 Pixels & $0.000 \%$ \\
\hline Low CE & $\mathrm{CE}<0.22 \%$ & 130 Pixels & $0.039 \%$ \\
\hline Unstable Dark Current & Change in $\mathrm{I}_{\mathrm{d}}>\mathbf{5 . 1 \times 1 0 ^ { 5 } \mathrm { e } - / \mathrm { s } \text { over } 4 5 \text { minutes }}$ & 9 Pixels & $0.002 \%$ \\
\hline Unstable CE & Change in CE $>0.4 \%$ of mean over 45 minutes & 163 Pixels & $0.049 \%$ \\
\hline Total Non-functional Pixels & NEdT $>0.33 \mathrm{~K}$ & 177 Pixels & $0.054 \%$ \\
\hline Total Functional Pixels & NEdT $\leq 0.33 \mathrm{~K}$ & 327503 Pixels & $99.85 \%$ \\
\hline Total Perfect Rows & Row containing 640 functional pixels & 401 Rows & $78.32 \%$ \\
\hline
\end{tabular}




\subsection{Pixel-to-pixel spectral uniformity}

It is desirable to have every pixel in the array exhibit the exact same spectral response. The conversion efficiency may increase or decrease by a uniform scale factor that can be easily corrected. However, a CE variation as a function of wavelength is undesirable. We refer to this $\mathrm{CE}$ variation as a function of wavelength as the spectral non-uniformity. Qualitatively, the spectral non-uniformity is the CE variation of an individual pixel from I $0.5 \mu \mathrm{m}$ to $I 2.3 \mu \mathrm{m}$ from the mean CE spectral response of that entire row of 640 pixels. To determine this spectral non-uniformity the mean spectral response at a given wavelength is computed for all 640 pixels in a given row. Figure 10 is a graph of the spectral response of two pixels in the same row (row 378) of ARL/GSFC 025 over-layed on the row mean spectral response from 7-15 $\mu \mathrm{m}$. Pixel 18 (near the edge of the array) exhibits a much greater spectral deviation from the mean than pixel 170 (near the center of the array).

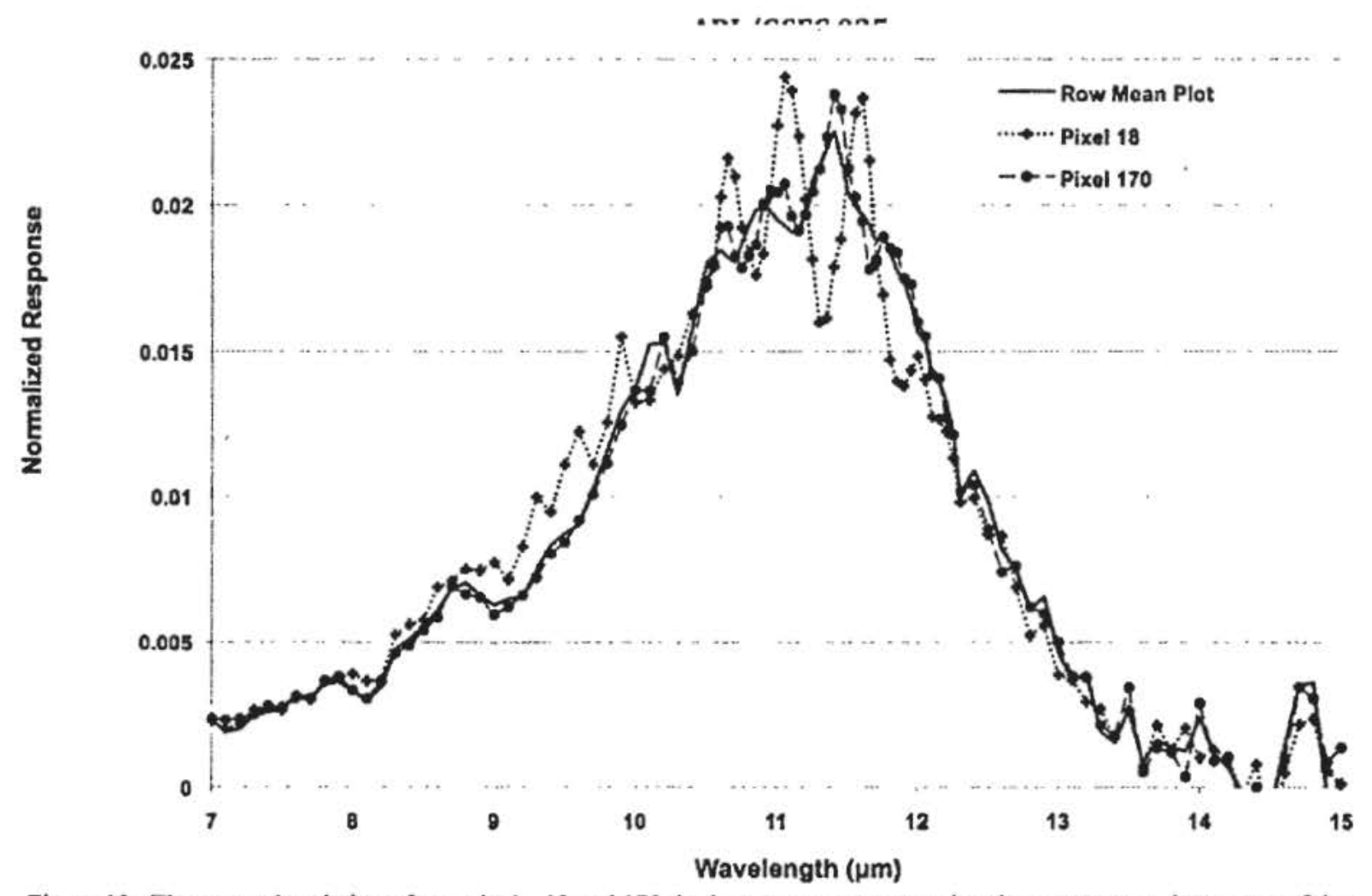

Figure 10. The spectral variation of two pixels, 18 and 170, in the same row compared to the mean spectral response of the entire row for ARL/GSFC $025 \mathrm{C}$-QWIP. It is clear that the pixel nearer to the edge of the array exhibits a higher degree of non-uniformity than the pixel well into the interior of the array for this fabrication process.

For the TIRS qualification and testing the spectral sampling starts at $10.50 \mu \mathrm{m}$ and is then stepped in $0.05 \mu \mathrm{m}$ increments up to $12.30 \mu \mathrm{m}$. Fifty frames are collected at each wavelength and averaged to give a single mean frame. The response of all 640 pixels in a given row is then averaged for a given wavelength. The first pixel response in the row corresponding to column 1 (which is also the average value of the 50 frames for that pixel) is then compared to the row mean. For every pixel in a given row the data set of percent deviation from the row mean versus wavelength is generated. However the goal is to distill this distribution into a single value so that the variation of the entire wavelength spectrum for each pixel can be compared to the mean of the entire spectrum of that row. This is the standard deviation (or noise) of the set of values given by:

$$
\mathbf{H}_{\mathbf{j}}=\left[\Sigma\left(\mathrm{w}_{\lambda_{\mathrm{n}}} *\left(\mathrm{p}_{\mathrm{j}, \lambda_{n}}-R_{\lambda_{n}}\right)^{2}\right) / \Sigma \mathrm{w}_{\lambda_{\mathrm{n}}}\right]^{1 / 2}
$$


where;

$\mathbf{H}_{\mathrm{j}}$ is the weighted pixel response standard deviation from the $\mathrm{i}^{\text {th }}$ row mean in the 10.5-12.3 $\mu \mathrm{m}$ band.

$\mathrm{w}_{\lambda \mathrm{n}}$ is a weighting factor that is equal to 1.0 except at 10.5 and $12.3 \mu \mathrm{m}$ where it is reduced to .25 (to mitigate the effect of the decreasing spectral response at the band edges).

$\mathrm{P}_{\mathrm{j}, \lambda \mathrm{n}}$ is the relative response of the $\mathrm{j}^{\text {th }}$ pixel in row $\mathrm{i}(\mathrm{i}=1 \ldots 512 ; \mathrm{j}=1 \ldots .640)$ at wavelength, $\lambda_{\mathrm{n}}$.

$\boldsymbol{R}_{\lambda \mathrm{n}}$ is the $\mathrm{i}^{\text {th }}$ row average relative response at $\lambda_{\mathrm{n}}$.

The spectral non-uniformity as a function of the pixel location in a given row is illustrated in figure 11. It is clear that arrays with different designs and different fabrication processes exhibit a characteristic non-uniform spectral response. Ideally, a flat line on the abscissa would be achieved. This is clearly not the case. The QmagiQ array is almost entirely below $5 \%$ whereas the ARL/GSFC array shows a characteristic increase in non-uniformity toward both ends of the row. The Thales array exhibits some asymmetry with the right half side of the array more spectrally non-uniform than the left side of the array. Since the spectral non-uniformity performance is not an overriding concern to the TIRS science no effort was expended to understand or improve the QmagiQ and Thales devices. However, some effort was made to determine the origin and possible the remedy for the ARL/GSFC C-QWIP spectral non-uniformity in a parallel side effort. Since all the arrays on a given wafer displayed similar nonuniformity characteristics the epitaxial growth process was determined not to be the cause. The fabrication a C-QWIP and a grating-based QWIP are dramatically different. The C-QWIP relies on a v-groove to delineate the pixels and for the optical coupling to the pixels After a few experiments we determined that by adjusting our etching parameters and modifying the array design at the periphery of the array we were able to substantially reduce this nonuniformity (with the value well below $6 \%$ in some rows). We attribute this improvement to a more uniform material removal at the array periphery. Other potential processing suspects which have not yet been studied include the epoxy underfill uniformity, device thinning nonuniformity and affects from the final reactive ion etch and chemical polishing.

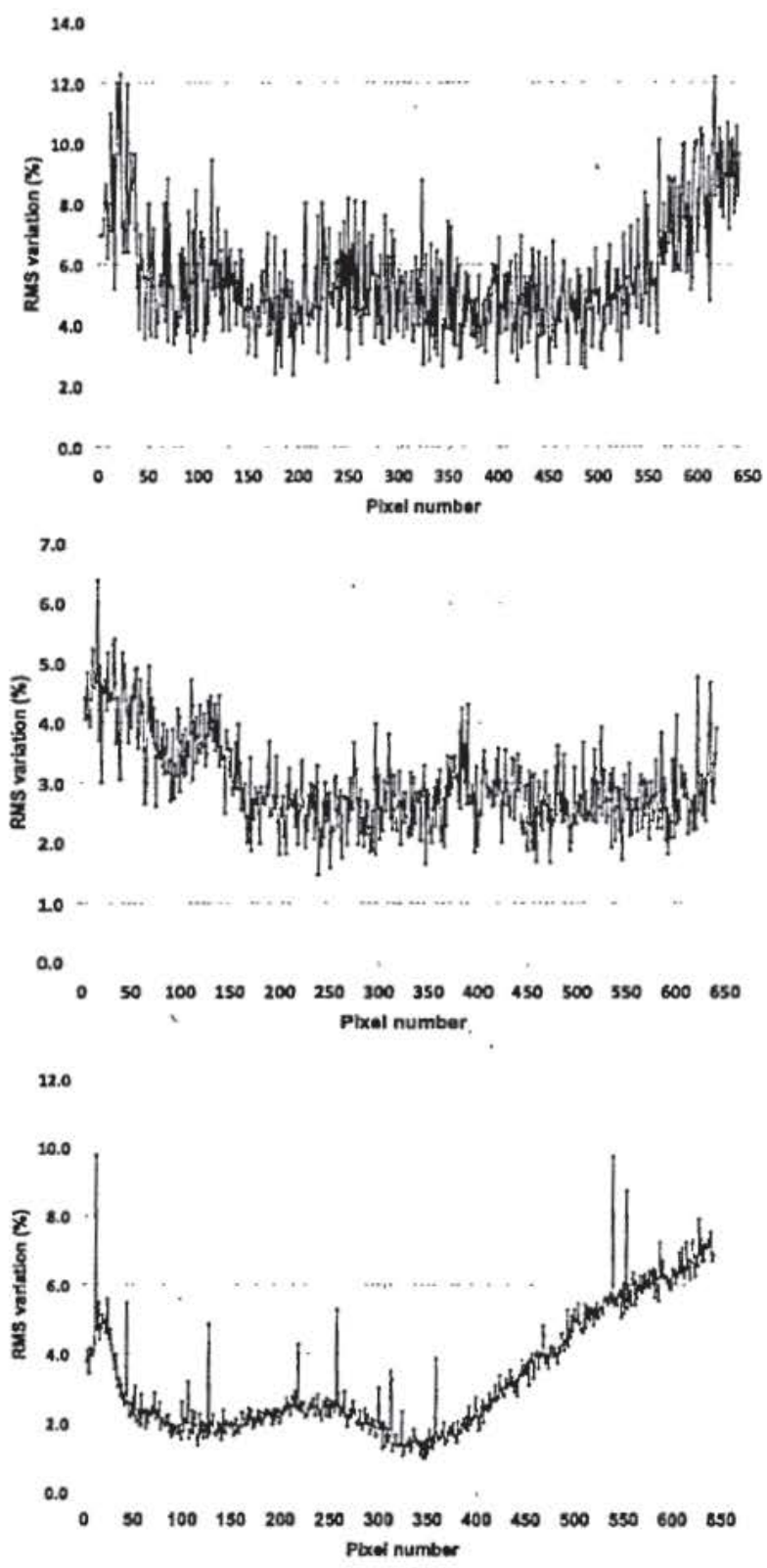

Figure 11. The spectral non-uniformity of each pixel in one row of the arrays compared to the row mean. The graph illustrates the composite spectral non-uniformity from 10.5 to $12.3 \mu \mathrm{m}$ of a given pixel compared to the mean spectral response of the entire row. 


\section{CONCLUSION}

A summary of the QWIP detector NE $\Delta T$ for each of the arrays fabricated by the different laboratories (QmagiQ Q020, ARL/GSFC 025 and Thales GT037) is shown in Table 3. The calculation is based on the difference between a $300 \mathrm{~K}$ and $301 \mathrm{~K}$ signal, that is a $\Delta \mathrm{T}=1 \mathrm{~K}$. The noise is calculated for each detector based on the dark current and CE. The read noise, operating temperature and integration time is assumed to be the same for all the arrays. However, the

Table 3. QWIP Array NEAT Based on the TIRS Parameters

\begin{tabular}{|c|c|c|c|}
\hline Parameter & QmagiQ & ARL/GSFC & Thales \\
\hline Temperature & $43 \mathrm{~K}$ & $43 \mathrm{~K}$ & $43 \mathrm{~K}$ \\
\hline Detector bias & $0.8 \mathrm{v}$ & $4.8 \mathrm{v}$ & $0.98 \mathrm{v}$ \\
\hline Photoconductive gain, $\mathrm{g}$ & 0.3 (assumed) & 0.22 (measured) & 0.27 (measured) \\
\hline Dark current, $I_{d}$ & $8 \mathrm{E} 7 \mathrm{e} / \mathrm{s}$ & $4 \mathrm{E} 8 \mathrm{e} / \mathrm{s}$ & $5 \mathrm{E} 7 \mathrm{e} / \mathrm{s}$ \\
\hline Integration time, $\mathrm{t}$ & $5.5 \mathrm{~ms}$ & $5.5 \mathrm{~ms}$ & $5.5 \mathrm{~ms}$ \\
\hline Peak $\lambda$ & $11.0 \mu \mathrm{m}$ & $11.3 \mu \mathrm{m}$ & $11.2 \mu \mathrm{m}$ \\
\hline Average CE near peak $\lambda(10.5-11.5 \mu \mathrm{m})$ & 0.023 & 0.018 & 0.022 \\
\hline Measured ROIC read noise, $\mathrm{l}_{\mathrm{r}}$ & $260 \mathrm{e}-$ & $260 \mathrm{e}-$ & 260 e- \\
\hline $301 \mathrm{~K}$ signal from source, $\mathrm{I}_{\mathrm{s}}(10.5-11.5 \mu \mathrm{m})$ & $8.9381 \mathrm{E} 10 \mathrm{ph} / \mathrm{s}$ & $8.9381 \mathrm{E} 10 \mathrm{ph} / \mathrm{s}$ & $8.9381 \mathrm{E} 10 \mathrm{ph} / \mathrm{s}$ \\
\hline $300 \mathrm{~K}$ signal from source, $\mathrm{I}_{\mathrm{s} 0}(10.5-11.5 \mu \mathrm{m})$ & $8.8073 \mathrm{E} 10 \mathrm{ph} / \mathrm{s}$ & $8.8073 \mathrm{EI} 0 \mathrm{ph} / \mathrm{s}$ & $8.8073 \mathrm{E} 10 \mathrm{ph} / \mathrm{s}$ \\
\hline $\begin{array}{l}\text { QWIP output signal for } 1 \mathrm{~K} \text { change in source T (TIRS } \\
\text { optical system, } 300 \mathrm{~K} \text { source; } 10.5-11.5 \mu \mathrm{m}), \mathrm{S} \\
\mathrm{S}=\mathrm{t} \cdot \mathrm{CE} \cdot(8.9381 \mathrm{E} 10-8.8073 \mathrm{E} 10)\end{array}$ & $1.65 \mathrm{E} 5 \mathrm{e}-$ & $1.29 \mathrm{E} 5 \mathrm{e}-$ & $1.58 \mathrm{E} 5 \mathrm{e}-$ \\
\hline Total noise contribution, $\mathrm{N}:\left[2 \mathrm{gt}\left(\mathrm{I}_{\mathrm{D}}+\mathrm{CE} \cdot \mathrm{I}_{\mathrm{S}}\right)+\mathrm{I}_{\mathrm{r}}{ }^{2}\right]^{1 / 2}$ & $2.65 \mathrm{E} 3 \mathrm{e}-$ & 2.I7E3e- & $2.43 \mathrm{E} 3 \mathrm{e}-$ \\
\hline Average NE $\Delta T$ at $\lambda_{\text {peak }},(N / S)$ & $16.1 \mathrm{mK}$ & $16.8 \mathrm{mK}$ & $15.4 \mathrm{mK}$ \\
\hline
\end{tabular}

detector bias voltage and photoconductive gain varies for each array. None of the arrays had an anti-reflective coating. It is apparent from the bottom line that the NE $\mathrm{T}$ performance of each array is remarkably similar. Based on the data presented throughout this paper it is clear that each of these arrays more than meets the LDCM/TIRS mission requirements and that the ultimate instrument performance is limited by parameters unrelated to the QWIP arrays. In other words, the arrays more than meet the requirements, by a substantial margin. This will prove to be of value as the instrument is finally assembled and tested since there may be instances where performance specifications are not being met by other subsystems and some relief may be available by decreasing the QWIP array performance but still remaining within specification. Two examples are; 1) if the focal plane was unable to reach the $43 \mathrm{~K}$ operating temperature, this would mean a higher dark current that could be offset by a reduction in detector bias or, 2) perhaps maintaining a focal plane temperature stability of $10 \mathrm{mK}$ proves to be infeasible, again lowering the dark current would mitigate this shortfall.

From the outset of the Landsat Data Continuity Mission it was clear that in order to meet schedule, cost and ensure reliability there would be very little margin for research and development. The QWIP technology proved to be uniquely capable of fulfilling this need and not with just one supplier but with multiple suppliers. Historically, QWIP technology has been relegated to playing a "niche" role and has not been seriously considered as a mainstream solution to LWIR technology challenges. However, this paradigm should shift and QWIP-based devices should be considered for all infrared applications and only eliminated in the instances where they absolutely cannot meet the mission requirements. If, for example, a far infrared system were required to capture every potential photon from a source, at whatever the cost, then one would consider using the absolutely most sensitive detector. For higher background applications this is 
generally not the case (in stark contrast to many astronomy missions). In the time span of 18 months, over 60 hybrids were developed from design through comprehensive performance testing yielding many flight candidates. Three separate laboratories all successfully produced flight quality devices and all meet the NASA Technology Readiness Level 6 requirements. This is partly the result of a dedicated team but in large part to the simplicity, suitability and reliability of the QWIP technology. This cannot be over-emphasized.

\section{Acknowledgements}

The authors would like to acknowledge the following individuals for their invaluable support to this NASA Landsat/TIRS project: Tom Hartman, Larry Hess, Audrey Ewin, Ron Hu, Nick Costen, Fred Wang, Steve Snodgrass, Allen Lunsford, Ed Wassel, Duncan Kahle, Bob Rosenberry, Greg Delo, Betsy Pugel, Sam Moseley, Avery Miles, Carol Sappington, Laddawan Miko, Trang Nguyen, Trinh Degumbia, Dennis Reuter, Jay Cho, Bing Guan, Phil Goodwin, Sherry Warner of NASA's Goddard Space Flight; Jason Bundas, Axel Reisinger, Rich Dennis, Kelly Patnaude, Doug Burrows, of QmagiQ, LLC and we would also like to express our appreciation to Jim Woolaway and Susan Petronio of Indigo Corp., Paul Pinsukanjana of Intelliepi, Corp. and Dave Hartzell of IQE Corp. for their ongoing support.

\section{References}

[I] See http://landsathandbook.gsfc.nasa.gov/handbook/handbook_htmls/chapter I/chapterl.html

[2] Jhabvala, M., Reuter, D., Choi, K., Sundaram, M., Jhabvala, C., Waczynski, A. and Bundas, J., SPIE, Orlando, FL, April, 2010.

[3] User's Guide ISC9803 Standard 640 Advanced Readout Multiplexer, version 1.2.

[4] Jhabvala, M., "Applications of GaAs QWIPs at the NASA/Goddard Space Flight Center", Infrared Physics and Technology, Vol. 42, June, 2001.

[5] Reuter, D., et al" The Thermal Infrared Sensor On The Landsat Data Continuity Mission", one of 22 authors, IGARSS, 2010. 